\title{
Post-Impact Hydrothermal Activity in Meteorite Impact Craters and Potential Opportunities for Life
}

\author{
Christian Koeberl \\ Department of Geological Sciences, University of Vienna, Althanstrasse \\ 14, A-1090 Vienna, Austria, christian.koeberl@univie.ac.at \\ Wolf Uwe Reimold \\ Impact Cratering Research Group, School of Geosciences, University of \\ the Witwatersrand, Johannesburg 2050, South Africa, \\ reimoldw@geosciences.wits.ac.za
}

\begin{abstract}
Impact craters are prominent landforms on all objects with a solid surface in the solar system. The formation of an impact structure is a high-energy, high-temperature transient event which introduces large amounts of energy into a limited area. This energy can, in part, be expanded on the creation and activation of a hydrothermal system within and even outside of the crater. Depending on the crater size and geology of the target region, this hydrothermal system may persist for extended periods of time. Such impact-induced hydrothermal systems could well have provided conditions supportive of the development of life on the early Earth and it is not unreasonable to assume that similar conditions could have led to life-favoring circumstances on other planets and satellites.
\end{abstract}

\section{Introduction}

Studies during the last few decades have convinced most workers that the planets formed by collision and hierarchical growth starting from small objects, i.e., from dust to planetesimals to planets. All planets and satellites of the solar system that have solid surfaces (e.g., Mercury, Venus, Mars, the asteroids) are covered by craters. Impact cratering has been either the most important, or one of the most important processes that affected the shaping of their surfaces. The Earth has had a violent impact-related history. Not only does the currently favored hypothesis for the origin of the Moon involve collision of the proto-Earth with a Mars-sized body, but also there is abundant evidence for a much higher impact flux at $\geq 3.8 \mathrm{Ga}$. It is known that the Moon experienced an interval of intense bombardment peaking at $\sim 3.85 \pm 0.05 \mathrm{Ga}$; it follows that the early Earth must have been subjected to an even more intense impact flux due to its larger size and because of its proximity to the Moon.

The rate at which the Earth is struck is fairly well understood. For example, asteroids with diameters $\geq 1 \mathrm{~km}$ collide with the Earth at a frequency of about 5 impacts per 1 million years, and each such impact forms a crater about 5-15 km in diameter. Impactors about $2 \mathrm{~km}$ in diameter collide with the Earth about 
every $1-2 \times 10^{6}$ years. Impacts of Earth-orbit crossing asteroids dominate the formation of craters on Earth that are smaller than about $30 \mathrm{~km}$ in diameter, whereas comet impacts probably caused the formation of the majority of craters larger than about $50 \mathrm{~km}$ in diameter.

Impact cratering is a high-energy event that occurs at more or less irregular intervals (although over long periods of time, an average cratering rate can be established). The recognition of the remnants of impact events is hampered by terrestrial processes (erosion, plate tectonics, sedimentation, etc.) that either cover or erase the surface expression of impact structures on Earth. In some cases, only remnants of distal ejecta have been found far from any possible impact structure. The study of such ejecta may lead, in turn, to the discovery of some impact craters (see for example Koeberl 2001).

Impact cratering has also influenced the geological and biological evolution of our planet. Even the impact of relatively small asteroids or comets can have disastrous consequences for our civilization. The chance that a large asteroid or comet $2 \mathrm{~km}$ in diameter (crater diameter $25-50 \mathrm{~km}$ ) may collide with the Earth during the next century is only 1 in 10000 . This could lead to severe disruption of the ecosphere and annihilation of a large percentage of Earth's population A better understanding of impact structures, their formation processes, and their consequences clearly has implications beyond planetary science, as discussed below, including consequences for life on Earth.

\section{Impact-induced Hydrothermal Systems}

Any impact event on Earth, as well as on the other terrestrial planets with an atmosphere (e.g., Mars), will generate a crater that represents a disruption of the hydrosphere. Impacts on land will affect not only target rocks of continental crustal composition, but also water, which is abundant on the Earth's surface; impacts into marine environments will directly affect the ocean. Even impacts into arid environments will potentially result in release of volatiles through shock dissociation of minerals. Interaction of aqueous solutions and other volatile components with hot, shocked rocks will result in hydrothermal activity, leading to effective alteration processes and potentially large-scale hydrothermal overprint on shock metamorphosed rocks, as well as deposition of secondary minerals from hydrothermal solutions. Several factors that control impact-induced hydrothermal activity can be distinguished (Naumov 2002): a) the formation of a long-term (in large craters, many thousands years) thermal anomaly, causing convection of ground and meteoric water; b) creation of an extensive zone of fractured rocks, favorable to the circulation of solutions and providing effective traps for mineralization from solution; c) effective and widespread shock deformation of mineral structures, which stimulates reactions between minerals and active fluids. As a result, hydrothermal alteration/mineralization is a common process in impact structures.

The size and duration of such a hydrothermal system will critically depend on the magnitude of the impact event, the amount of deposited kinetic energy, the rock volume affected, the mineralogical composition of the rock types present and their suitability for chemical interaction with solutions, and the amount of volatiles released/set into circulation. The cooling processes involved will 
determine the duration of the active hydrothermal process. Low-temperature hydrothermal processes may even provide environments for the generation of biologically active environments, perhaps creating unique niches for new development of life. Even in small impact craters, crater lakes often develop relatively shortly after dissipation of impact energy and initial cooling of the system. Impact craters form relatively closed systems that may draw their lake water either from artesic systems in the crater floor or from collection of precipitation (or both).

In a number of craters, the hydrothermal mineralization has been studied in some detail, mainly as a result of drilling projects (Naumov 2002). Only in a few cases was it possible to study the three-dimensional distribution of post-impact hydrothermal mineralization and to construct a reliable model of a post-impact circulation system. In this respect, the three large impact craters in Russia Popigai,Kara, and Puchezh-Katunki - provided some of the best information available, due to the detailed investigations, including drilling, carried out at these locations. Naumov (2002) demonstrated that the hydrothermal mineral associations in many terrestrial impact craters - smectites, chlorites, zeolites, calcite, anhydrite, epidote, pyrite, and quartz - are similar.

Post-impact hydrothermal systems were studied at, for example, the Roter Kamm, Ries, Manson, Haughton Dome, and Siljan impact structures (Newsom 1980; Newsom et al. 1986; Koeberl et al. 1989; Osinski et al. 2001). After the impact event, which leads to vaporization and melting of large amounts of country rock, a hydrothermal system can be maintained for several tens of thousands of years. Crater lakes and subsurface water systems can provide a warm and wet environment relative to the environs of the crater structure. Heat sources can be the volumes of uplifted rocks in the central region of a large, complex impact structure, as well as the bodies of hot impact breccia filling parts of the interior of impact structures.

\section{Distribution of the Hydrothermal Mineralization in Impact Struc- tures}

The hot-water circulation system in and around the central uplift area, as well as local convective cells in impact melt bodies, is determined by the post-impact thermal anomaly in an impact crater. After Naumov (2002), two areas, the central and peripheral areas of the post-impact hydrothermal alteration, can be distinguished, which differ not only in their location within the structures, but also in the type and distribution of hydrothermal minerals.

The central area includes authigenic breccia (the central uplift of shocked basement rocks) and overlying suevite and lithic breccia, as well as possible crater lake deposits in the central parts of a crater. Both a vertical and a less well-defined lateral zonation of the hydrothermal mineralization is a main characteristic of this area; the Puchezh-Katunki structure is an example. Similar features of the vertical zonation of hydrothermal alteration have been found in other craters where deep drilling in central uplifts was carried out: Ries, Boltysh, Siljan Ring, and Manson (e.g., McCarville \& Crossey 1996). The temperature of crystallization of hydrothermal minerals decreases upward as well as outward 
from the crater center; temperature intervals of mineral crystallization in these zones are estimated to be $180-350^{\circ} \mathrm{C}$ and $50-200^{\circ} \mathrm{C}$, respectively.

The peripheral area includes impactites and impact breccia that fill the annular depression around a central uplift. At Popigai and Kara (Naumov 2002), hydrothermal mineralization in the peripheral area includes the entire annular depression, where a number of low-temperature minerals, similar to the upper zone of the central area, occur: smectites, chlorites, zeolites, calcite, pyrite. The basic features of the distribution of the hydrothermal mineralization here are the almost complete absence of any zonation and the constant chemical compositions and crystal structures of secondary minerals. A vertical zonation characterized by the presence of different phyllosilicates within thick impactite sequences is found in same cases, indicating the existence of thermal gradients during the initial stage of the hydrothermal activity.

\section{Characteristics of Post-Impact Hydrothermal Solutions}

The composition of hydrothermal mineralization indicates that surface, meteoric, and ground water (and, possibly, products of shock dehydration and degassing of minerals) are the sources for hot aqueous, post-impact solutions. This is indicated by fluid inclusion data (e.g., Koeberl et al. 1989) and by measurements of the isotopic composition of oxygen and hydrogen from some hydrothermal minerals in impact structures (e.g., Komor \& Valley 1990). Shocked target rocks are sources for the elements contained in such solutions. Mineral associations typical for vapor-dominated hot-water circulation systems are absent in impact craters. The general succession of mineral crystallization in all environments confirms the uniformly regressive course of the hydrothermal process. The conditions for the formation of hydrothermal minerals in craters vary within a narrow interval of $\mathrm{pH}$ and correspond to weakly alkaline and near-neutral environments $(6<\mathrm{pH}<8)$, mainly as a consequence of the leaching of host rocks, which consist mainly of shock-derived aluminosilicates and impact-generated glasses. The solutions maintain Eh values of $>-0.5 \mathrm{~V}$ (for a neutral environment) in the course of the hydrothermal process.

\section{Development of an Impact-Generated Hydrothermal System}

Naumov (2002) proposed that the hydrothermal process in large impact structures can be subdivided into three successive stages: 1) an initial stage, when the isotherms preserve their original configuration (the temperature decreases from top downward); 2) a main stage after an inversion of the thermal field; the thermal gradients during this stage range from about $30^{\circ} \mathrm{C} \mathrm{km}^{-1}$ in the central part of the hydrothermal cell to $100^{\circ} \mathrm{C} \mathrm{km}^{-1}$ on its periphery; and 3) a final stage, during which thermal gradients are less than $10-30^{\circ} \mathrm{C} \mathrm{km}^{-1}$ and hot-water circulation takes place in near-surface areas only. The vertical paragenetic zonation, which imitates a deep geothermal source, results from the thermal evolution of an impact structure. The creation of a vertical zonation of the distribution of hydrothermal minerals in central uplifts belongs to the main stage when the circulation system involves the maximum amount of rock. Finally, zeolite-calcite cement of redeposited suevite and lithic breccia, as well as 
latest-stage calcite veins, form during the final stage. Thermal contribution from impactites filling the crater may create local thermal anomalies with gradients insignificant to provide zonation of the hydrothermal alteration. Nevertheless, thick impact melt rock sequences occurring in large impact structures can cause local thermal anomalies during the early modification stage.

\section{Conclusions and Implications for Life}

The long-term result of any impact-generated hydrothermal activity is expressed mainly in the formation of secondary mineral assemblages. Mineralogical and geochemical features of impact-generated hydrothermal processes are governed by a combination of both chemical composition of the target and conditions of the host rock as a result of shock and thermal metamorphism. The products of impact-generated hydrothermal processes are similar to the hydrothermal mineralization in volcanic areas, as well as in modern geothermal systems, because thermodynamic and hydrodynamic conditions are similar. Some distinctive features of the impact-induced hydrothermal mineralization exist, though, as described by for example Naumov (2002). These include defects in the crystal structures of minerals, enrichment of zeolites and phyllosilicates due to the availability of free silica, low gas content in fluid inclusions, etc. Work is planned by us for the near future to carry out a deep drilling project at the Bosumtwi impact crater in Ghana, which contains a large crater lake and which may have provided a warm post-impact oasis for the recolonization of the area (cf. Osinski et al. 2001), possibly leading to the development of endemic species. Thus, impact events are not only destructive events (i.e., responsible for mass extinctions), but can also have positive effects on the development of life on Earth and, possibly, other planets.

In terms of importance of impact events for the development of life, only few studies exist. Newsom et al. (1986) were among the first to postulate that impact-induced hydrothermal systems in Martian craters could have provided life-sustaining environments. Cockell \& Lee (2002) reviewed data from the Haughton Dome impact structure (Arctic Canada) regarding recolonization of an impact structure (see also Osinski et al. 2001). However, a lot more work on this topic is necessary. On the early Earth, the availability of hot or warm niches undoubtedly must have had a major influence on the development of early life. Similar processes may have operated on other planets, especially Mars, and it is conceivable that the Jovian satellite Europa has also benefited from the existence of hydrothermal systems. There is no reason to assume that planets around other stars would not have been - and still are - subject to impact events; impact cratering is a process that must be assumed to be operative in the entire universe and that must have caused results similar to those on Earth or other terrestrial planets, on other planetary systems as well.

Acknowledgments. Supported by the Austrian Science Foundation (Y58GEO). University of the Witwatersrand Impact Cratering Research Group Contribution No. 65. 


\section{References}

Cockell, C., \& Lee, P. 2002, Biological Reviews 77, 279

Koeberl, C. 2001, in Accretion of Extraterrestrial Matter throughout Earth's History, The sedimentary record of impact events, ed. B. PeuckerEhrenbrink \& B. Schmitz, (Kluwer Academic/Plenum Publishers), 333

Koeberl, C., Fredriksson, K., Götzinger, M., \& Reimold, W.U. 1989, Geochimica et Cosmochimica Acta 53, 2113

Komor, S. C., \& Valley, J. W. 1990, Contributions to Mineralogy and Petrology, 105,516

McCarville, P., \& Crossey, L. J. 1996, in The Manson Impact Structure, Iowa: anatomy of an impact crater, Geological Society of America Special Paper 302, ed. C. Koeberl \& R. R. Anderson, 347

Naumov, M. A. 2002, in Meteorite Impacts in Precambrian Shield, Impact Studies vol. 2, ed. J. Plado \& L. Pesonen, (Heidelberg-Berlin: Springer), 117

Newsom, H. E. 1980, Icarus, 44, 207

Newsom, H. E., Graup G., Sewards, T., \& Keil, K. 1986, in Proc. of Lunar and Planetary Sci. Conf. 17th, Journal of Geophysical Research, 91, 239

Osinski, G. R., Spray, J. G., \& Lee, P. 2001, Meteor. and Planet. Sci., 36, 731 